\section{Epidemiology of the Association between Anticoagulants and Intraocular Hemorrhage in Patients with Neovascular Age-Related Macular Degeneration}

\author{
Daniel F. Kiernan, MD ${ }^{1}$ \\ Seenu M. Hariprasad, $\mathrm{MD}^{2}$ \\ Irene M. Rusu, BA ${ }^{2}$ \\ Sahil V. Mehta, BS ${ }^{2}$ \\ William F. Mieler, MD ${ }^{1}$ \\ Rama D. Jager, $\mathrm{MD}^{2,3}$
}




\section{Summary Statement}

The cumulative and annual incidence of developing subretinal or vitreous hemorrhage is significantly higher in patients with neovascular AMD who are taking daily aspirin, clopidogrel or warfarin compared to patients not taking these agents. Use of these medications poses a significantly greater odds ratio for developing intraocular hemorrhage than other possible risk factors including age, gender, diabetes, hypertension and bilateral neovascular AMD. 


\begin{abstract}
Purpose: To determine the cumulative and annual incidence of intraocular hemorrhage (subretinal hemorrhage or vitreous hemorrhage) in patients with neovascular age-related macular degeneration (neovascular AMD) and association with daily antiplatelet or anticoagulant (AP/AC) medication usage (aspirin, clopidogrel and warfarin), age, gender, hypertension, diabetes mellitus or bilateral neovascular AMD.
\end{abstract}

Design: Retrospective, cross-sectional study in a tertiary University setting.

Methods: Data on one hundred and ninety-five eyes of 195 patients without prior intraocular hemorrhage examined over seventy-three months was reviewed.

Results: Ninety-six of 195 (49.2\%) patients were taking daily AP/ACs. 63.5\% of patients taking daily $\mathrm{AP} / \mathrm{AC}$ agents had hemorrhage compared to $29.2 \%$ of patients not taking $(\mathrm{OR}=4.21,95 \% \mathrm{CI}=1.42-8.46, \mathrm{p}<0.001)$. The overall annual incidence of intraocular hemorrhage was $0.14 \%$ per year. Among patients taking daily AP/AC, the cumulative incidence $(61 / 96,63.5 \%)$ and annual incidence $(0.10 \%)$ of concurrent intraocular hemorrhage was significantly greater compared to patients not taking them $(29 / 99,29.2 \%$ and $0.04 \%$, respectively, $\mathrm{p}<0.0001)$. Fourteen of $18(77 \%)$ patients taking more than one daily AP/AC had occurrence of intraocular hemorrhage. AP/AC usage was an independent risk factor for the development of intraocular hemorrhage. The use of any agent resulted in a significantly increased risk of developing intraocular hemorrhage. Additionally, presence of bilateral neovascular AMD was a significant association in 
those taking daily $\mathrm{AP} / \mathrm{ACs}$, whereas age was a significant association in those not taking daily $\mathrm{AP} / \mathrm{AC}$ agents.

Conclusions: All three daily AP/AC types were significantly associated with an increased risk of the development intraocular hemorrhage in patients with neovascular AMD, whereas gender, hypertension and diabetes were not. Age was not significantly associated with hemorrhage in patients taking daily $\mathrm{AP} / \mathrm{AC}$ agents whereas the presence of bilateral neovascular AMD was. These findings indicate that the AP/AC use may predispose neovascular AMD patients to intraocular hemorrhage more so than age and duration of disease alone. While the risk that discontinuing these medicines would pose to the patients' health may be too great to justify, ensuring that an appropriate medication dosage is maintained should be a priority within this patient population. 


\section{Introduction}

The percentage of Americans over the age of 60 years continues to rise, from $16.2 \%$ in 2000 to $16.6 \%$ in $2006 .{ }^{1,2}$ Life expectancy has also risen steadily to an average of 80.4 years for women and 75.2 years for men. ${ }^{3}$ However with increasing age, the prevalence of chronic diseases such as coronary artery disease, atherosclerosis and hypertension is also increasing, as is the use of medications designed to decrease mortality, such as antiplatelet (AP) and anticoagulant (AC) agents. ${ }^{4-6}$ Ophthalmologists have also become increasingly aware of potential problems associated with the use of these medications, including hemorrhagic complications, occurring in association with a variety of multispecialty surgical procedures, ${ }^{7-11}$ including vitrectomy surgery and intravitreal injections. $^{12-13}$

There is also evidence that patients with age-related macular degeneration (AMD) who are taking AP or AC medications may be at increased risk for hemorrhagic complications including severe submacular or vitreous hemorrhage associated with choroidal neovascularization (CNV). When hemorrhage enters the subretinal space anterior to Bruch's membrane, it causes photoreceptor destruction secondary to iron toxicity, blockage of nutrient diffusion and clot retraction, disciform scar formation, and may lead to permanent visual loss. ${ }^{14-18}$ Surgical management of this situation has resulted in variable outcomes, but is generally associated with a poor visual prognosis and a high rate of surgery-associated complications. ${ }^{19-22}$ Given that the number of Americans with AMD is expected to grow to more than 12 million by the year $2020,{ }^{23}$ and the prevalence of AP and AC usage is also likely to rise with an increasing elderly population, the question of whether these agents are independently associated with an 
increased incidence of intraocular hemorrhage in neovascular AMD patients becomes increasingly important. There have been a limited number of studies examining the role of AP/ACs in patients with neovascular $\mathrm{AMD}^{14-17}$ and there have been no studies to determine if clopidogrel bisulfate (Plavix, Bristol-Myers Squibb, New York, NY) is associated with an increased intraocular bleeding risk in patients with neovascular AMD. Furthermore, although it seems intuitive that there should be an increased incidence of hemorrhagic complications in this patient population, there are no epidemiologic studies reporting these data in the ophthalmic literature.

Therefore, we sought to determine the cumulative and yearly incidence of intraocular hemorrhage in patients with neovascular AMD and if daily aspirin, clopidogrel or warfarin sodium (Coumadin, Bristol-Myers Squibb, New York, NY) is an associated risk factor. In addition, we looked for any correlation with other possible risk factors including age, gender, and the presence of hypertension, diabetes mellitus or bilateral neovascular AMD.

\section{Materials and Methods}

University of Chicago IRB approval was obtained prior to carrying out this study (protocol No. 16161A). A retrospective analysis of two-hundred and fifty-six patient charts identified with the International Statistical Classification of Diseases-9 (ICD-9) code 362.52 "exudative senile macular degeneration of retina" was performed for every patient seen between January 2002 and January 2008 (73 months). Inclusion criteria included a history of neovascular AMD and all current medications documented on clinical examination and verified through primary care medical record. Exclusion criteria 
included any prior intraretinal hemorrhage, lack of documented neovascular disease, history of trauma, posterior segment surgery or neovascularization in the study eye not related to neovascular AMD. In patients without prior intraocular hemorrhage, the first occurrence of clinically evident subretinal hemorrhage within one disk diameter of the fovea or vitreous hemorrhage anywhere in one eye was reviewed and recorded ( 1 eye per patient only) as was photographic and angiographic evidence of neovascular AMD and subretinal hemorrhage, in addition to clinical information including total follow-up period, age, gender, presence of diabetes, hypertension or bilateral neovascular disease and use of $\mathrm{AP} / \mathrm{AC}$ at all examinations prior to and at the time of hemorrhage. For patients taking $\mathrm{AP} / \mathrm{AC}$ at the time of hemorrhage, follow-up duration was calculated based on the first recorded active clinical medication list that included an AP/AC. Medical records were also reviewed to determine the international normalized ratio (INR) values for those taking warfarin. Statistic analysis including multivariate logistic regression (Stata version 8.2, Stata Corp. College Station, TX) was performed in order to determine significance $(\mathrm{p}<0.05)$, odds ratio (OR) for developing hemorrhage and $95 \%$ confidence intervals (CI) based on the risk factors of total follow-up period, age, gender, presence of diabetes, hypertension, bilateral neovascular disease or use of AP/AC. Baseline factors, including age, gender, and the presence of diabetes, hypertension or bilateral neovascular disease was compared between groups using Student's t-test (Stata version 8.2).

\section{Results}

From the 256 charts identified, one hundred and ninety-five patients met entry criteria, and were included in this study. Sixty-one charts were excluded for the following 
reasons or diagnoses: no documented neovascular disease $(n=25)$, prior posterior segment surgery $(n=13)$, myopic degeneration $(n=5)$, prior pan-retinal photocoagulation treatment for proliferative diabetic retinopathy $(n=5)$, presumed ocular histoplasmosis syndrome $(n=3)$, polypoidal vasculopathy $(n=3)$, prior trauma $(n=2)$, central retinal vein occlusion $(n=1)$, Sorsby's fundus dystrophy $(n=1)$, angioid streaks $(n=1)$, ocular toxoplasmosis $(n=1)$ and serpiginous choroiditis $(n=1)$.

Ninety-six of 195 patients were taking daily AP/ACs (49.2\%) during a median followup of 27.0 months (range, 1-73 months). The average INR was 2.2 (range 1.2-2.9). Baseline factors which were similar between patients taking or not taking AP/AC agents included median follow-up (28.0 and 21.5 months, $\mathrm{p}=0.10)$, age, gender, presence of hypertension, and presence of bilateral neovascular disease. However, there were significantly more diabetic patients taking AP/ACs compared to those not taking them (Table 1).

Ninety-one patients $(46.6 \%)$ had an occurrence of intraocular hemorrhage over the 6.08 year study period, meaning that among patients with neovascular AMD, the annual incidence was $0.14 \%$. Among patients taking daily $\mathrm{AP} / \mathrm{AC}$, the cumulative incidence $(61 / 96,63.5 \%)$ and annual incidence $(0.10 \%)$ of concurrent intraocular hemorrhage was significantly greater compared to patients not taking them $(29 / 99,29.2 \%$ and $0.04 \%$, respectively, $\mathrm{p}<0.0001)$. In addition, hemorrhage incidence density was 0.022 and 0.013 per patient-month, for those taking or not taking AP/AC respectively. Fourteen of 18 (77\%) patients taking more than one daily AP/AC had occurrence of intraocular hemorrhage. Multivariate logistic regression analysis demonstrated that AP/AC usage, age and presence of bilateral neovascular AMD were independent risk factors for 
hemorrhage and had significant odds ratios (Table 2). Seven patients had an occurrence of massive vitreoretinal hemorrhage and 6 of these patients were on one or more AP/AC therapies, implying that the annual incidence of combined subretinal and vitreous hemorrhage was $0.009 \%$ in patients using daily AP/ACs and $0.001 \%$ in patients not taking them.

\section{Discussion}

The overall cumulative incidence, annual incidence and incidence density of intraocular hemorrhage in patients with neovascular AMD is significantly higher in those taking daily oral $\mathrm{AP} / \mathrm{AC}$ medications. Although the population in this study is one already at risk for ocular hemorrhage by having neovascular AMD, ${ }^{24}$ the overall cumulative $(63.5 \%)$ and annual incidence $(0.10 \%)$ of hemorrhage is much less compared to prior reports of major or minor systemic hemorrhagic complications $(1.2-7 \%$ and 2 $24 \%$, respectively) in patients receiving long-term anticoagulant therapy. ${ }^{25-26}$ It is also less than in another study which reported that $3 \%$ of patients, without ocular disease, who were taking warfarin had incidental retinal hemorrhage on dilated eye examination. ${ }^{27}$ The percentage of patients taking $\mathrm{AP} / \mathrm{ACs}$ is greater in the present study than in a metaanalysis of 68 patients with hemorrhagic complications associated with AMD reported in the literature from 1965 to 1985 in which $19-27 \%$ of patients were taking various nonsteroidal anti-inflammatory AP drugs, including aspirin, or ACs. ${ }^{16}$ Another report found that $23 \%$ of patients with intraocular hemorrhage were taking either AP or AC agents and that AMD patients with massive combined vitreous and subretinal hemorrhages were 11.6 times more likely to be taking warfarin compared to AMD patients presenting with 
small subretinal hemorrhage, ${ }^{14}$ which is comparable to the OR for developing hemorrhage reported in the present study of 10.7 for overall hemorrhage risk. Although no patients were taking clopidogrel, the same previous study described a non-statistically significant odds ratio of 2.1 for aspirin in patients with massive intraocular bleeding, ${ }^{14}$ whereas our results indicate significant associations of both aspirin and clopidogrel with intraocular hemorrhage. This underscores the importance for ophthalmologists to inquire about specific $\mathrm{AP} / \mathrm{AC}$ agents and to communicate with the medical specialist about the patient's AMD status.

This is the first study to report the cumulative incidence and annual incidence of vitreous hemorrhage in patients with neovascular AMD. Six of the 7 patients presenting with these massive hemorrhages were on AP or AC therapy. Although uncommon, the visual consequences of such hemorrhages can be devastating and surgical intervention has limited visual outcomes. ${ }^{19-22}$ Future analysis of other predisposing conditions in addition to $\mathrm{AP} / \mathrm{AC}$ usage may help identify and develop prophylactic treatment for this uncommon, yet devastating complication of AMD.

In patients not using daily $\mathrm{AP} / \mathrm{ACs}$, increased age was the only associated risk factor for intraocular hemorrhage. Age is a strongly associated risk factor for the development of $\mathrm{CNV}$ in patients with $\mathrm{AMD}^{28}$ and subsequent fibrotic scarring may form a cleavage plane for the development of submacular hemorrhage. ${ }^{14-16,29}$

The increased risk of hemorrhage associated with patients taking AP/ACs who also had bilateral neovascular AMD in this study may indicate that predisposing systemic reasons for using these agents, such as cardiovascular disease, peripheral arterial disease, deep venous thrombosis, increased serum cholesterol or other factors which were not 
analyzed may play a role in the pathogenesis of AMD and hemorrhage. The fact that significantly more patients who were taking AP/ACs had diabetes also indicates that this condition may have contributed toward the medical indication for these agents.

An Age-Related Eye Disease Study report reported a higher incidence of central geographic atrophy associated with use of anti-inflammatory medications including aspirin. ${ }^{30}$ Our data show that patients taking daily aspirin, the most common antiplatelet agent in this and other major studies ${ }^{5,31}$ were 3.75 times more likely to develop a subretinal hemorrhage than patients not taking it. Although the role of aspirin in decreasing mortality associated with myocardial infarctions and cerebral vascular accidents is well-established, ${ }^{31-32}$ its role as an anti-inflammatory agent and platelet inhibitor in the pathogenesis of AMD remains unclear.

The effect of confounding sources of bias was minimized by using multivariate regression analysis. Misclassification bias was minimized by careful review of medical records and fundus photographs for each subject. Selection bias may have influenced our results since the study was performed at a tertiary university-based referral center, where incidence of intraocular hemorrhage is relatively high, especially since these patients may have been referred for management of their condition.

Another weakness of this study is its retrospective nature; a randomized, prospective cohort study would have greater power to estimate the relative risk of individual AP or $\mathrm{AC}$ medications since the cumulative incidence of intraocular hemorrhage is relatively high. However, the reduced risk of myocardial infarction among patients taking aspirin compared to controls in a previous study would ethically preclude randomization of this agent due to excess cardiovascular risk among the placebo group. ${ }^{33}$ It would be equally 
difficult to justify halting the clopidogrel or warfarin that patients may be taking for lifethreatening conditions as that may significantly increase patient mortality.

Although intraocular hemorrhage complicating AMD is not as directly life-threatening as major complications of anticoagulant use which necessitate hospitalization and blood transfusion, it can have devastating consequences on the remaining visual potential, quality of life and expenditures of an elderly patient. This has important implications for such therapy initiation and maintenance among AMD patients. Patients with a history of AMD who are at greater risk of intraocular hemorrhage, such as those starting warfarin or an additional agent to one they are already taking should undergo an ophthalmic evaluation with dilated fundus exam at the initiation of treatment. Patients who are identified by their ophthalmologist as being at a higher risk for ocular hemorrhagic complications, especially those with neovascular disease in both eyes, may need to have more frequent follow-up with dilated fundus examinations. Concurrent use of AP/ACs may also have an impact on the decision to increase the frequency of intravitreal injection treatments, since regression of subclinical CNV should decrease the likelihood of subretinal or vitreous hemorrhage from occurring. While the risk that discontinuing these medicines would pose to the patients' health may be too great to justify, the potential interactions with other drugs and ensuring that an appropriate medication dosage and a therapeutic INR (for those on warfarin) is maintained should be discussed with the patient and other physicians and health care staff responsible for patient care. 


\section{Acknowledgements}

The authors have no financial, commercial or propriety interest in any of the products mentioned herein. Thank you to Matthew Georgopulos and Chitra Radhakrishnan for their editorial suggestions on this manuscript. Presented at the 2009 Combined Meeting of the Retina Society, Macula Society and the American Society of Retina Specialists, New York, NY. Supported by Research to Prevent Blindness.

\section{REFERENCES}

1. From: 2006 United States Census data:

http://www.census.gov/population/www/popdata.html. Last accessed September 12, 2009.

2. From: 2000 United States census data: http://www.census.gov/Press-

Release/www/2001/demoprofile.html. Last accessed September 12, 2009.

3. From: 2005 the United States Centers for Disease Control, life expectancy:

http://205.207.175.93/HDI/TableViewer/tableView.aspx?ReportId=169. Last accessed September 12, 2009.

4. Jneid H, Bhatt DL. Advances in antiplatelet therapy. Expert Opin Emerg Drugs 2003;8:349-363.

5. Lutsep HL. Recent clinical trials of antiplatelet therapy in secondary stroke prevention: expectations and results for application in primary care settings. Am J Med. 2009 Apr;122(4 Suppl 2):S21-31.

6. Angiolillo DJ, Bhatt DL, Gurbel PA, Jennings LK. Advances in antiplatelet therapy: agents in clinical development. Am J Cardiol. 2009 Feb 2;103(3 Suppl):40A-51A.

7. Barequet IS, Sachs D, Priel A, et al. Phacoemulsification of cataract in patients 
receiving Coumadin therapy: ocular and hematologic risk assessment. Am J Ophthalmol. 2007 Nov;144(5):719-723.

8. Benzimra JD, Johnston RL, Jaycock P, et al; EPR User Group. The Cataract National Dataset electronic multicentre audit of 55,567 operations: antiplatelet and anticoagulant medications. Eye. 2009 Jan;23(1):10-6.

9. Law SK, Song BJ, Yu F, et al. Hemorrhagic complications from glaucoma surgery in patients on anticoagulation therapy or antiplatelet therapy. Am J Ophthalmol. 2008 Apr;145(4):736-746.

10. Dayani PN, Grand MG. Maintenance of warfarin anticoagulation for patients undergoing vitreoretinal surgery. Trans Am Ophthalmol Soc. 2006;104:149-60.

11. Parkin B, Manners R. Aspirin and warfarin therapy in oculoplastic surgery. Br J Ophthalmol. 2000 Dec;84(12):1426-7.

12. Dayani PN, Siddiqi OK, Holekamp NM. Safety of intravitreal injections in patients receiving warfarin anticoagulation. Am J Ophthalmol. 2007 Sep;144(3):451-3.

13. Meyer $\mathrm{CH}$, Eter N. Incidence of ocular hemorrhages in anticoagulated patients receiving repeated intravitreal injections. Am J Ophthalmol. 2008 Feb;145(2):386-7.

14. Tilanus MA, Vaandrager W, Cuypers MH, Verbeek AM, Hoyng CB. Relationship between anticoagulant medication and massive intraocular hemorrhage in age-related macular degeneration. Graefes Arch Clin Exp Ophthalmol. 2000 Jun;238(6):482-5.2.

15. Lewis H, Sloan SH, Foos RY. Massive intraocular hemorrhage associated with anticoagulation and age-related macular degeneration. Graefe's Arch Clin Exp Ophthalmol 1988;226:59-64. 
16. El Baba J, Jarett WH II, Harbin TS Jr, et al. Massive hemorrhage complicating agerelated macular degeneration. Clinicopathological correlation and role of anticoagulation. Ophthalmology 1986;93:1581-1592.

17. Weir C, Nolan DJ, Holding D, Hammer H. Intraocular haemorrhage associated with anticoagulant therapy. Acta Ophthalmol Scand 2000;78:492-493.

18. Toth CA, Morse LS, Hjelmeland LM, Landers MB III. Fibrin directs early retinal damage after experimental subretinal hemorrhage. Arch Ophthalmol 1991;109:723-729.

19. Hattenbach LO, Klais C, Koch FJ, Gümbel HOC. Intravenous injection of tissue plasminogen activator and gas in the treatment of submacular hemorrhage under various conditions. Ophthalmology 2001;108:1485-1492.

20. Giansanti F, Eandi CM, Virgili G. Submacular surgery for choroidal neovascularisation secondary to age-related macular degeneration. Cochrane Database Syst Rev. 2009;(2):CD006931.

21. MacLaren RE, Uppal GS, Balaggan KS, et al. Autologous transplantation of the retinal pigment epithelium and choroid in the treatment of neovascular age-related macular degeneration. Ophthalmology. 2007;114(3):561-70.

22. Hochman MA, Seery CM, Zarbin MA. Pathophysiology and management of subretinal hemorrhage. Surv Ophthalmol 1997;42:195-213.

23. Friedman DS, O'Colmain BJ, Muñoz B, et al. Prevalence of age-related macular degeneration in the United States. Arch Ophthalmol 2004;122:564-72.

24. Watzke RC (1988): Acquired macular disease. In: Duane TD. Jaeger EA ed. Clinical Ophthalmology; Philadelphia: Harper \& Row, Vol. 3. Chap 23. pp. 3-4. 
25. Schulman S. Clinical practice. Care of patients receiving long-term anticoagulant therapy. N Engl J Med. 2003;349(7):675-83.

26. Levine MN, Raskob G, Landefeld S, Kearon C. Hemorrhagic complications of anticoagulant treatment. Chest 2001;119: Suppl:108S-121S.

27. Superstein R, Gomolin JE, Hammouda W, et al. Prevalence of ocular hemorrhage in patients receiving warfarin therapy. Can J Ophthalmol. 2000 Dec;35(7):385-9.

28. Complications of Age-related Macular Degeneration Prevention Trial (CAPT) Research Group. Risk factors for choroidal neovascularization and geographic atrophy in the complications of age-related macular degeneration prevention trial. Ophthalmology. 2008 Sep;115(9):1474-9.

29. Wolter JR, McWilliams JR. Rupture of diciform macular degeneration causing massive retroletinal hemorrhage. Am J Ophthalmol. 1965 Jun;59:1044-7.

30. Clemons TE, Milton RC, Klein R, Seddon JM, Ferris FL 3rd; Age-Related Eye Disease Study Research Group. Risk factors for the incidence of Advanced Age-Related Macular Degeneration in the Age-Related Eye Disease Study (AREDS) AREDS report no. 19. Ophthalmology. 2005 Apr;112(4):533-9.

31. Wolff T, Miller T, Ko S. Aspirin for the primary prevention of cardiovascular events: an update of the evidence for the U.S. Preventive Services Task Force. Ann Intern Med. 2009 Mar 17;150(6):405-10.

32. Hegge KA. Antiplatelet agents for prevention of recurrent ischemic stroke. S D Med. 2009 Jan;62(1):15-7.

33. Christen WG, Glynn RJ, Ajani UA, et al. Age-related maculopathy in a randomized trial of low-dose aspirin among US physicians. Arch Ophthalmol 2001;119:1143-1149. 


\section{TABLES}

Table 1: Baseline patient demographics.

Table 2: Multivariate analysis of risk factors. 


\begin{tabular}{|c|c|c|c|}
\hline Demographic & $\mathbf{N}$ & $\%$ & $\begin{array}{l}\text { t-test } \\
\text { p-value }\end{array}$ \\
\hline Gender (Male/Female) & $79 / 116$ & $41 / 59$ & 0.54 \\
\hline On AP/AC & $41 / 55$ & $43 / 57$ & \\
\hline Not on AP/AC & $38 / 61$ & $38 / 62$ & \\
\hline Age (years) & 83.1 & 100 & 0.08 \\
\hline On AP/AC & 84.8 & 49.3 & \\
\hline Not on AP/AC & 82.1 & 50.7 & \\
\hline AP/AC & 96 & 49.2 & --- \\
\hline Aspirin & 80 & 41.0 & \\
\hline Clopidogrel & 18 & 9.2 & \\
\hline Warfarin & 16 & 8.2 & \\
\hline Multiple & 18 & 9.2 & \\
\hline Hypertension & 97/195 & 49.7 & 0.69 \\
\hline On AP/AC & $45 / 96$ & 46.8 & \\
\hline Not on AP/AC & $52 / 99$ & 52.5 & \\
\hline Diabetes mellitus & 29/195 & 14.8 & 0.02 \\
\hline On APIAC & $20 / 96$ & 20.8 & \\
\hline Not on AP/AC & $9 / 99$ & 0.9 & \\
\hline Bilateral wet AMD & $106 / 195$ & 54.6 & 0.42 \\
\hline On AP/AC & $55 / 96$ & 57.2 & \\
\hline Not on AP/AC & $51 / 99$ & 51.5 & \\
\hline
\end{tabular}

Abbreviations: OR, odds ratio; $\mathrm{Cl}$, confidence interval; AP/AC, antiplatelet or anticoagulant; AMD, age-related macular degeneration. 


\begin{tabular}{|c|c|}
\hline Independent Variable & Odds Ratio $(95 \% \mathrm{Cl})$ \\
\hline $\begin{array}{l}\text { Gender } \\
\text { On AP/AC } \\
\text { Not on AP/AC }\end{array}$ & $\begin{array}{l}1.68(0.88-3.21) \\
1.46(0.58-3.70) \\
1.82(0.72-4.57)\end{array}$ \\
\hline $\begin{array}{l}\text { Age } \\
\text { On AP/AC } \\
\text { Not on AP/AC }\end{array}$ & $\begin{array}{l}1.05(1.00-1.09) \\
1.03(0.97-1.10) \\
1.05(1.00-1.11)\end{array}$ \\
\hline $\begin{array}{l}\text { APIAC } \\
\text { Aspirin } \\
\text { Clopidogrel } \\
\text { Warfarin } \\
\text { Multiple }\end{array}$ & $\begin{array}{l}4.21(2.15-8.26) \\
3.75(1.88-7.48) \\
8.76(2.32-32.9) \\
10.7(2.45-46.6) \\
12.9(3.00-55.4)\end{array}$ \\
\hline $\begin{array}{l}\text { Hypertension } \\
\text { On AP/AC } \\
\text { Not on AP/AC }\end{array}$ & $\begin{array}{l}1.08(0.53-2.22) \\
0.76(0.22-2.56) \\
1.41(0.56-3.58)\end{array}$ \\
\hline $\begin{array}{l}\text { Diabetes mellitus } \\
\text { On AP/AC } \\
\text { Not on AP/AC }\end{array}$ & $\begin{array}{l}\mathbf{0 . 6 2}(\mathbf{0 . 2 4}-\mathbf{1 . 6 0}) \\
0.82(0.26-2.59) \\
0.35(0.03-3.35)\end{array}$ \\
\hline $\begin{array}{l}\text { Bilateral wet AMD } \\
\text { On AP/AC } \\
\text { Not on AP/AC }\end{array}$ & $\begin{array}{l}1.96(1.04-3.70) \\
3.47(1.42-8.46) \\
1.08(0.42-2.74)\end{array}$ \\
\hline
\end{tabular}


WORK(S) COVERED UNDER THIS AGREEMENT: This agreement includes all submitted written material as well as any supplementary digital material including but not limited to audio, video, and other data files whose formats may vary.

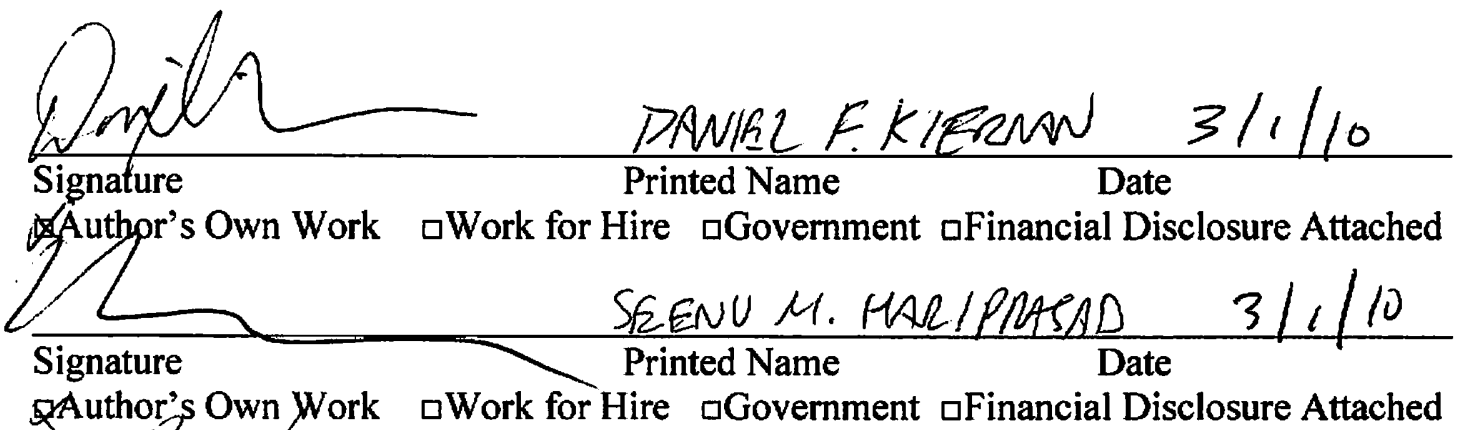
Author's Own Work $\square$ Work for Hire $\quad$ Government $\square$ Financial Disclosure Attached
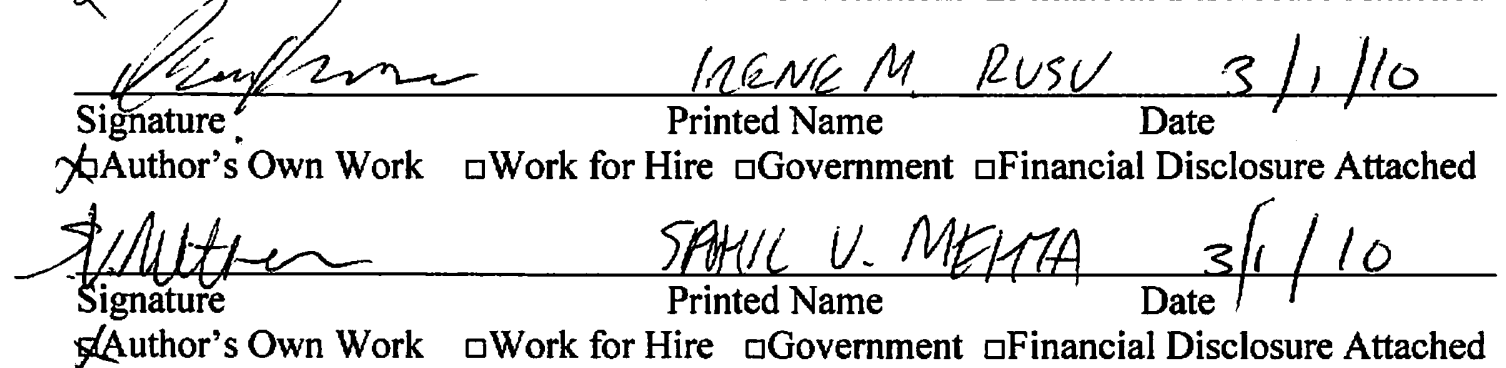

\& Author's Own Work $\square$ Work for Hire $\quad$ Government $\square$ Financial Disclosure Attached

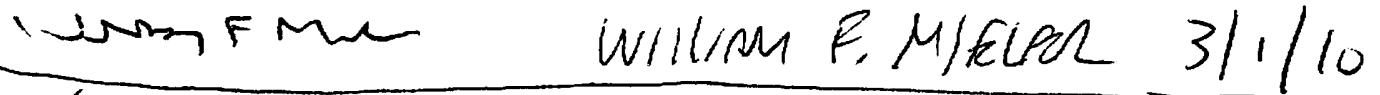

Q Driven's OWn work

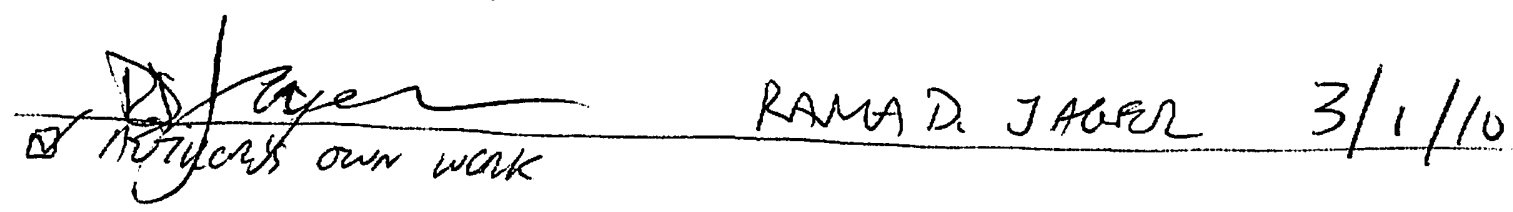

